\title{
WDM-PON Preventive Optical Monitoring System with Colourless Reflectors
}

\author{
J. Montalvo ${ }^{(1)}$, A. Tapetado ${ }^{(2)}$, D.S. Montero ${ }^{(2)}$, C. Vázquez ${ }^{(2)}$ \\ (1) Telefónica Investigación y Desarrollo, S.A.U., Ronda de la Comunicación, s/n, 28050 Madrid (Spain) \\ (2) Universidad Carlos III de Madrid, Avda. Universidad 30, 28911, Leganés, Madrid (Spain) \\ Author e-mail address: julio.montalvogarcia@telefonica.com
}

\begin{abstract}
A new optical monitoring system for remote measurement of fibre attenuation in WDMPONs is reported. It uses colourless reflectors at ONU side, avoiding inventory complexity. Its performance is demonstrated with negligible penalty on $12.5 \mathrm{~Gb} / \mathrm{s}$ transmission.
\end{abstract}

OCIS codes: (060.2300) Fiber measurements; (060.2330) Fiber optics communications; (280.4991) Passive remote sensing; (120.0280) Remote sensing and sensors.

\section{Introduction}

During latest years, massive deployments of fibre access networks are taking place worldwide very rapidly, already surpassing 100 million Fibre to the Home (FTTH) subscribers in 2014 [1]. While residential deployments are in their vast majority supported by power splitter based fibre infrastructures and GPON/EPON standards, Wavelength Division Multiplexing Passive Optical Networks (WDM-PONs) based on Wavelength Routed Optical Distribution Networks (WR-ODN) can be a cost-effective solution when logical point-to-point connections are required for higher security and performance, such as aggregation networks, mobile front-hauling or business services. In these scenarios, especial monitoring tools are needed in order to reduce operational costs of the optical distribution network and to guarantee high Service Level Agreement (SLA) requirements.

While remote optical transceiver monitoring [2] using in-band diagnostics can be helpful for remotely checking the optical parameters in the customer premises equipment (CPE), this approach offers a low measurement accuracy $( \pm 3 \mathrm{~dB})$ and is only available when the device in the customer premises is active and working properly. As a consequence, the remote diagnostic of optical transceivers does not allow preventive ODN monitoring and cannot distinguish between fibre and CPE faults in some situations. Recently, Tunable Optical Time Domain Reflectometers (T-OTDRs) [3] have been proposed overcoming the former restrictions, with the capability of performing in-service fibre monitoring and fault localization in the drop fibres of WDM-PONs independently of CPEs operation.

As an optional complement to OTDR systems, a preventive low cost technique for quick fibre monitoring in Coarse WDM-PONs has also been proposed [4], but it requires two coloured reflectors at each branch of the PON and cannot be used with the network in service.

In this paper, we propose a new low-cost preventive monitoring system for Dense WDM-PONs which allows inservice measurement and uses a single colourless reflector at ONU side, thus reducing the number of components required and avoiding complexity.

\section{Architecture of the measurement technique}

The generic architecture of the proposed technique is shown in Fig. 1.(a). The proposed architecture can be applied to a general scenario where an Access/Aggregation node, typically a WDM-PON Optical Line Termination (OLT), is connected to several remote devices, typically Optical Network Units (ONUs), through a point-to-multipoint WRODN. This communication system may be a FTTH/B network, as well as aggregation and mobile backhaul/fronthaul networks, where the node in the operator premises may be a Multi-Protocol Label Switching (MPLS) based metro Ethernet aggregation switch, and remote devices may be OLTs, Digital Subscriber Line Access (DSLAMs) or Base Stations (BS) of a mobile network.

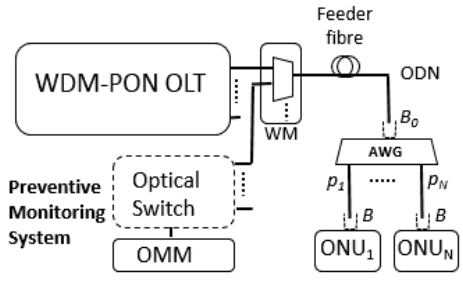

(a)

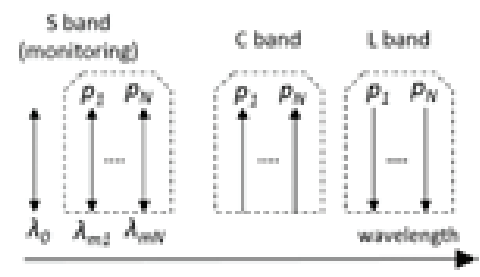

(b)

Fig. 1. (a) General architecture of the proposed technique. OMM: Optical Monitoring Module. AWG: Arrayed Waveguide Grating. (b) Waveband plan for the in-service preventive monitoring system. 
In the network operator premises, the monitoring system consists of a centralized optical monitoring module (OMM), an optional optical switch to select the desired ODN to be tested, and an array of wavelength multiplexers (WM) that combines the data signals from each optical port of the Access/Aggregation Node with the test signal of the OMM. In the ODN, the technique adds a single reflector $B_{0}$ in the input of a cyclic Arrayed Waveguide Grating (AWG) mux/demux with $N$ ports $\left(p_{1} \ldots p_{N}\right)$. Finally, at the end of each branch of the mux/demux (or optionally inside the remote devices/ONUs), a single colourless optical reflector $B$ is used.

\section{Principle of operation}

The monitoring technique can operate at the same time than data transmission within the same ODN. In the OMM, a reference optical signal $\left(\lambda_{0}\right)$ and a monitoring broadband light source (BLS) are delivered to the WR-ODN through the corresponding WM. Both reference and monitoring signals are comprised within free wavebands in the ODN not used for data transmission. The reference optical signal is reflected by reflector $B_{0}$ at the input of the AWG and received back in the OMM. On the other hand, the monitoring broadband light source is filtered by the AWG, delivering a different monitoring signal $\left(\lambda_{m l}-\lambda_{m N}\right)$ to the end of the corresponding AWG output port $\left(p_{1} \ldots p_{N}\right)$, where the monitoring signal is reflected by reflector $B$ and received back in the OMM. The reflectance waveband of all reflectors $B$ covers all the monitoring waveband, thus all the reflectors are identical (colourless). This monitoring waveband covers one cycle of the AWG which is not used by the data signals, see Fig. 1.(b). At the same time, for each port of the AWG data signals are transmitted upstream and downstream using other cycles of the AWG in the C- and L-bands, thus each AWG port is simultaneously used for data transmission and monitoring.

For each remote device, the reflections of the reference signal $\left(\lambda_{0}\right)$ and the monitoring signal $\left(\lambda_{m i}, i=1 \ldots N\right)$ are digitally processed in the OMM, and self-referenced measurement values are obtained. The measurement values obtained depend on the optical losses at the AWG for each port and the fibre attenuation between each AWG port and each colourless reflector $(B)$ of the corresponding remote device.

\section{Self-referencing parameters}

For each ODN branch $i(i=1 \ldots N)$, a self-referenced measurement parameter $\phi_{i}$ is obtained [5], which is calculated as the electrical phase shift between the reflected waves at $\lambda_{0}$ and $\lambda_{m i}$ received at the OMM. For each branch, the sensitivity to the fibre attenuation and the linearity of the self-referencing parameters can be configured in a flexible way using phase shifting in the reference and monitoring reflected signals.

Because of the self-referencing nature of the signal processing at the OMM, the measured values are resilient to variations of the absolute optical power and sensitivity of the light sources and receivers, respectively. The use of Radio-frequency (RF) modulation in the monitoring signals allows achieving improved Signal-to-noise-ratio (SNR) in the measurements, which are also insensitive to the interference of other electronic signals.

\section{Experimental validation}

A Bit Error Rate (BER) tester using DWDM SFP+ transceivers is used to emulate OLT ports and ONUs, see Fig. 2. The OMM is formed by an electro-optical (EO) stage and a general purpose platform (GPP), running a virtual signal processing software. The computer code consists of four stages: data acquisition, filtering, phase shifter and lock-in amplifier. The software takes 8 seconds in acquiring and processing the 32 channels of the AWG. More details of the OMM structure can be found in [5-6].

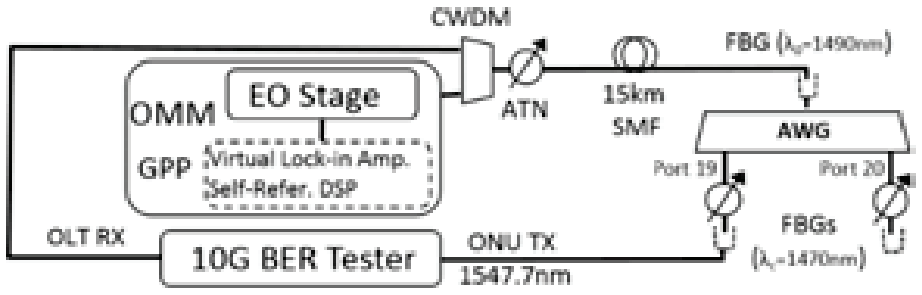

Fig. 2. Experimental set-up for validation of the principle of operation.

The ODN consists of a 15-km single-mode fibre (SMF) coil between the OLT port and a cyclic 32-port AWG with several cycles in the S-, C- and L-bands.

The S-band is used as monitoring waveband, while the C-and L-bands are used for data transmission between ONU and OLT.

A Fibre Bragg Grating with nominal reflectance centered at $1490 \mathrm{~nm}$ is used as reference reflector $B_{0}$. Two identical wideband Fibre Bragg Reflectors with nominal reflectances centered at 1470nm are used in output ports 19 and 20 of 
the AWG as reflectors $B$. Optical attenuators (ATN) are employed at each output port to emulate fibre attenuation of each ODN branch. In the experiments an attenuation range from $0 \mathrm{~dB}$ to $5.5 \mathrm{~dB}$ is configured for maximum linearity of the self-referenced parameters.

For each attenuation value at output ports 19 and 20 of the AWG, self-referenced parameters $\phi_{19}$ and $\phi_{20}$ are obtained in the OMM. The mathematical expression for $\phi_{i}$, see Eq. (7) in [5], is ruled by the fibre attenuation as well as the phase-shifts $\left(\Omega_{1}, \Omega_{2}\right)$ configured at the OMM. The monitoring system provides high-linearity or high-sensitivity monitoring performance, this flexibly is configured as desired.

In order to test the self-reference of the measurement technique, an optical attenuator adding optical losses up to $8 \mathrm{~dB}$ is used, with negligible variations $(0.2 \mathrm{~dB})$ of the measurement parameter $\phi_{19}$. A measurement accuracy of fibre attenuation as low as $0.27 \mathrm{~dB}$ is achieved, meanwhile $\mathrm{ONU}$ data transmission at $12.5 \mathrm{~Gb} / \mathrm{s}\left(\mathrm{NRZ}, \mathrm{PRBS}=2^{31}-1\right)$ is also active.

The self-referenced measurements of $\phi_{19}$ versus the attenuation of the ODN branch 19 are shown in Fig. 3.(a). A crosstalk test with regards the attenuation of ODN branch 20 can also be seen in the same figure, showing a negligible crosstalk $(<0.1 \mathrm{~dB})$ between the two self-referenced measurements in both AWG adjacent channels.

Finally, a maximum power penalty of $0.37 \mathrm{~dB}$ on data transmission due to the use of the optical monitoring technique has been obtained, see Fig. 3.(b).

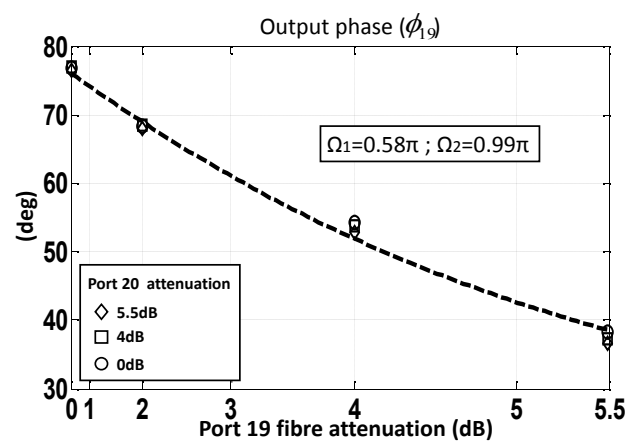

(a)

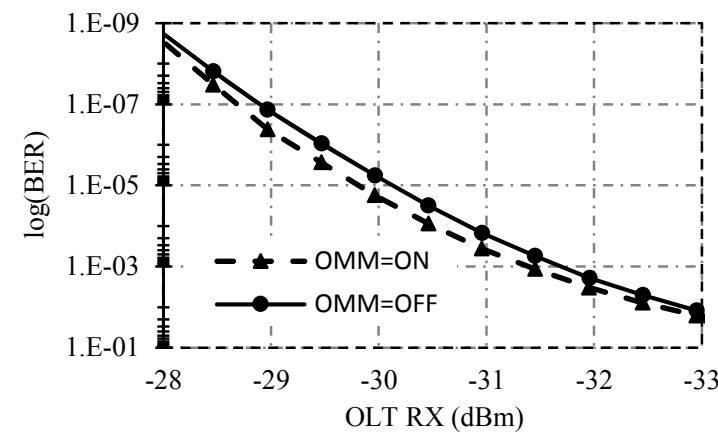

(b)

Fig. 3. (a) Self-referenced measurements $\phi_{l}$ versus AWG port 19 attenuation, crosstalk test versus AWG port 20 attenuation. (b) BER for ONU $12.5 \mathrm{~Gb} / \mathrm{s}$ transmission @ $1547.7 \mathrm{~nm}$ with $(\mathrm{OMM}=\mathrm{ON})$ and without monitoring system $(\mathrm{OMM}=\mathrm{OFF})$.

\section{Conclusions}

A new preventive optical monitoring system for remote self-referenced measurement of the drop fibre attenuation in WDM-PONs has been described and experimentally validated. An attenuation measurement accuracy of $0.27 \mathrm{~dB}$ with a maximum power penalty of $0.37 \mathrm{~dB}$ in a $12.5 \mathrm{~Gb} / \mathrm{s}$ transmission has been demonstrated using a general purpose platform. The setup is capable of measuring the attenuation of 32 fibers in 8 seconds.

\section{Acknowledgements}

The research leading to these results has received funding from the Spanish Ministry of Economía y Competitividad under projects TEC2012-37983-C03-02 (CFOOT-TIC) and TEC2009-14718-C03-03, from the Comunidad de Madrid project under grant S2013/MIT-2790 (SINFOTON-CM) and support from the European Union under grant no. 318137 (DISCUS).

\section{References}

[1] "FTTH, DSL, and Cable Subscribers report," Infonetics Research (2015).

[2] "Specification for Diagnostic Monitoring Interface for Optical Transceivers", SFF-8472 Rev. 12.2, SFF Committee (2014).

[3] D. Villafani et al., “Tunable OTDR Measurements for WDM-PON Monitoring,” Proc. IMOC, Rio de Janeiro (2013).

[4] J. Montalvo et al., "Radio-frequency self-referencing system for monitoring drop fibres in wavelength division multiplexing passive optical networks," IET Optoel., Vol. 4, no. 6, p. 226 (2010).

[5] A. Tapetado et al., "A self-referenced optical intensity sensor network using POFBGs for biomedical appliactions", Sensors, Vol. 14, p. 24029 (2014).

[6] C. Vázquez et al., "Método y sistema para la monitorización de redes de fibras ópticas”, ES, Patent application nº P201530018, (2015). 